Article

\title{
A Spatially Distributed Network for Tracking the Pulsation Signal of Flow Field Based on CFD Simulation: Method and a Case Study
}

\author{
Faye Jin ${ }^{1,2}$, Ran Tao ${ }^{1,2, *}$, Zhaoheng Lu ${ }^{1,2}$ and Ruofu Xiao ${ }^{1,2}$ \\ 1 College of Water Resources and Civil Engineering, China Agricultural University, Beijing 100083, China; \\ jfy@cau.edu.cn (F.J.); luzhaoheng@foxmail.com (Z.L.); xrf@cau.edu.cn (R.X.) \\ 2 Beijing Engineering Research Center of Safety and Energy Saving Technology for Water Supply Network \\ System, China Agricultural University, Beijing 100083, China \\ * Correspondence: randytao@cau.edu.cn
}

Citation: Jin, F.; Tao, R.; Lu, Z.; Xiao, R. A Spatially Distributed Network for Tracking the Pulsation Signal of Flow Field Based on CFD Simulation: Method and a Case Study. Fractal Fract. 2021, 5, 181. https://doi.org/ $10.3390 /$ fractalfract 5040181

Academic Editors: Carlo Cattani, Mustafa Inc and Mehmet Ali Akinlar

Received: 14 August 2021

Accepted: 19 October 2021

Published: 23 October 2021

Publisher's Note: MDPI stays neutral with regard to jurisdictional claims in published maps and institutional affiliations.

Copyright: (c) 2021 by the authors. Licensee MDPI, Basel, Switzerland. This article is an open access article distributed under the terms and conditions of the Creative Commons Attribution (CC BY) license (https:// creativecommons.org/licenses/by/ $4.0 /)$.

\begin{abstract}
The pulsating characteristics in turbulent flow are very important physical quantities. There are many studies focused on the temporal characteristics of pulsation. However, the spatial distribution of temporal states with pulsations rarely receives attention. Therefore, the pulsation tracking network (PTN) method is proposed to track the pulsating characteristics of turbulence. Based on the computational fluid dynamics (CFD) simulation result, the PTN is arranged in a specific region of the flow domain. The fast Fourier Transform (FFT) method is used for time-frequency conversion. As shown in the example of trailing-edge vortex-shedding flow over NACA0009 hydrofoil, important pulsation quantities, including the total pulsation intensity, dominant frequencies, amplitude of frequencies, and the phase and phase difference, can be obtained with a high spatial resolution. The source, reason and attenuation of the vortex-shedding frequency $f_{v s}$ and the $2 f_{v s}$ frequency caused by vortex-interaction are well indicated. The dominant regions of $f_{v s}$ and $2 f_{v s}$ are shown and analysed. The propagation and attenuation of vortex-shedding induced pulsation are understood in detail. Based on the comparison against traditional analysis, PTN is found to function as a good supplement for the CFD post-processing by tracking unknown temporal and spatial characteristics. These findings represent a potential breakthrough in terms of solving actual pulsation-excited flow problems.
\end{abstract}

Keywords: turbulent flow; time-frequency conversion; pulsation tracking; temporal spatial state; computational fluid dynamics

\section{Introduction}

Karman vortex street is an important phenomenon in viscous incompressible fluid dynamics. The study of Karman vortex street is usually based on the case of flow around a cylinder or a foil [1,2]. In recent years, the research methods of temporal and spectral characteristics of flow fields were gradually applied to unsteady flow fields [3,4]. The modal decomposition of flow fields can be divided into two categories: one is based on data-driven approaches (POD, SPOD and DMD). The second is based on operator-driven approaches (Koopman analysis, global linear analysis, stability analysis and resolvent analysis).

The former is based on flow field data, such as CFD calculation results and experimental measurements; The latter is based on the linearized N-S equation. However, its most obvious advantage is that it has no requirements for input, that is, this data can be linear or nonlinear, and the results can be numerical or experimental. This approach does not necessarily even involve the calculation results of a flow field. The essence of model reduction is to project the system into a smaller vector space that can describe the parameters.

The essence of POD is to find low dimensional subspaces (i.e., flow modes or coherent structures), and it expresses the high-dimensional and complex unsteady flow field as the 
superposition of these subspaces on the low dimensional coordinate system. The flow field evolution in the low dimensional space was previously described, and the dominant vortex structure in the unsteady flow field was identified [5]. Lumley [6,7] identified the coherent structure in a turbulent flow field by means of the orthogonal decomposition of a spatial velocity correlation function [8], which was called direct-POD. With the rapid development of experimental and numerical simulations, the original data that need to be de-composed is increasingly large and complex, which leads to the large dimensions of the flow field information matrix. The direct-POD method cannot solve its eigenvalues and eigenvectors. Sirovich [9] improved the direct-POD method and put forward the concept of space-time conversion. He used a time cross-correlation matrix instead of a space cross-correlation matrix and successfully solved the problem of too many space points. This method is called snapshot-POD. The dominant coherent structure distribution can be identified by sorting the proportion of each mode of energy [10,11]. The main characteristic of this method is the relationship between the reduced data and the original data, including the numerical relationship among the mean value, eigenvector and principal component [12]. However, the frequency analysis of the pulsating signal is slightly insufficient in the POD method.

SPOD (spectral proper orthogonal decomposition) expands the POD and was published by Towne [4] in 2018. POD decomposes the data into the eigenmode, eigenvalue (energy), etc. However, this evolution coefficient cannot be expressed as a specific function of time. Furthermore, the time series can be represented by a regular time function via the Fourier transform method. SPOD realizes the time-space conversion by converting the time domain to the frequency. SPOD expands the time orthogonality and retains all the characteristics of POD.

DMD involves the extraction of dynamic information from unsteady experimental measurement or numerical simulation flow fields, and was proposed by Schmid $[13,14]$. In essence, the flow evolution is regarded as a linear dynamic process. Through the characteristic analysis of the flow field snapshot of the whole process, the low-order modes and their corresponding eigenvalues (or Ritz values) are obtained. The most important feature of this method is that the decomposed mode has a single frequency and growth rate [15].Therefore, it has great advantages in the analysis of dynamic linear and periodic flow, but needs to occupy a lot of data storage space. Kunihiko et al. [16] described some of the main techniques used to complete these modal decompositions and analyses. These techniques have been booming in recent decades. Some mature techniques are briefly introduced, and the framework of these methods is clearly established by using familiar linear algebra. Pan et al. [17] applied DMD to study the wake velocity field of NACA0015 airfoil measured by TR-PIV. The spatial motion of vortex pairs usually shows the coupling of long wave and short-wave modes.

The parameters of the periodic signal mainly include the frequency, phase and amplitude [18]. The amplitude is directly obtained by analysing the sampled values in the time series (time domain analysis), but many problems require information about the pulsation frequency. In the frequency domain analysis, the distribution of signal strength relative to frequency is studied, which is expressed by the spectrum. The spectrum value is complex, the spectrum has real and imaginary parts, and each frequency has a phase angle [19]. The amplitude spectrum is the description of signal contour and shape. The phase spectrum is a description of the signal position. The amplitude spectrum of signals with the same shape is the same at different positions, but the phase spectrum is different $[20,21]$. In other words, the frequency reflects the frequency of the pulse. The amplitude indicates the intensity of the pressure pulsation. The phase represents the state of the signal at a specific time.

In this study, we introduce a pulsation tracking network (PTN) method for the tracking of turbulent pulsation characteristics. The frequency decomposition of the flow field pulsation signal is studied. This method allows for a better understanding of important parameters, including the amplitude, peak-to-peak value, dominant frequency, phase and phase difference, pulsation attenuation and pulsation propagation. A simple case 
of hydrofoil trailing-edge vortex-shedding flow is used as an example to show the main functions of PTN. Traditional analyses are also conducted for pressure pulsation to show the advantages of PTN. Innovative results will be shown in the following sections. The results will demonstrate that PTN could be a breakthrough and an effective supplement to CFD simulation, especially in post-processing.

\section{Temporal Spatial States of Pressure Pulsation}

Based on FFT, a periodic signal can be represented as the addition of different sinusoidal (or cosine) signals, and these sinusoidal (or cosine) signals are orthogonal. Through the integral Fourier Transform formula, the frequency and amplitude of these sinusoidal (or cosine) signals can be solved accurately. The frequency domain diagram can be obtained as follows:

$$
\begin{gathered}
f(t)=\frac{1}{2 \pi} \int_{-\infty}^{+\infty} F(\omega) \cdot e^{i \omega t} d \omega \\
F(\omega)=\int_{-\infty}^{+\infty} f(t) e^{-i \omega t} d t
\end{gathered}
$$

where $\omega=n \cdot \Delta \omega(n=1,2,3 \ldots)$, and $\Delta \omega$ can be considered as the frequency interval of the periodic function of adjacent frequencies.

Furthermore, the pulsation of pressure $\Delta P$ can be expressed as:

$$
\Delta P=P_{\max }-P_{\min }
$$

where $P_{\max }$ is the maximum value defined in the pressure pulsation, and $P_{\min }$ is the minimum value. In order to avoid the error caused by signal interference, a certain confidence interval (for example, $97 \%$ ) is considered when calculating the peak-to-peak value according to the IEC regulations [22].

For a certain frequency (the main frequency is shown here), the phase of a certain point is selected as the initial phase $\varphi_{0}$, and the phase at point $k$ is expressed as $\varphi_{k}$. The FFT characteristic frequency phase difference $\Delta \varphi_{k}$ can be calculated as follows:

$$
\Delta \varphi_{k}=\left|\varphi_{k}-\varphi_{0}-2 m \pi\right|
$$

where $\left\{\begin{array}{c}\varphi_{0}>0 \\ \varphi_{k} \leq \varphi_{0}-\pi\end{array}, n=-1 ;\left\{\begin{array}{c}\varphi_{0} \leq 0 \\ \varphi_{k} \leq \varphi_{0}+\pi\end{array}\right.\right.$ or $\left\{\begin{array}{c}\varphi_{0}>0 \\ \varphi_{k}>\varphi_{0}-\pi\end{array}, n=0\right.$;

$\left\{\begin{array}{c}\varphi_{0} \leq 0 \\ \varphi_{k}>\varphi_{0}+\pi\end{array}, n=1\right.$. The value of the $\Delta \varphi_{k}$ range is $0 \sim \pi$. The values of the $\varphi_{k}$ and $\varphi_{0}$ ranges are $-\pi \sim \pi$.

\section{Pulsation Tracking Network (PTN) Method}

\subsection{Estimated Distance of Adjacent Monitoring Points}

A large number of ordered monitoring points should be well distributed in the flow field to prepare for PTN. Thus, there is a necessary parameter, the estimated distance of adjacent monitoring points, denoted as $D_{E}$, which can be expressed as follows:

$$
D_{E}=\frac{c_{s} U_{r e f}}{2 \pi f_{\max }}
$$

where $U_{r e f}$ is a reference flow velocity, $\mathrm{m} / \mathrm{s} ; f_{\max }$ is the maximum main frequency in the monitoring area, $\mathrm{Hz} ; c_{S}$ is the safety factor, which controls the density of monitoring points and is used to refine the length of $D_{E}$ for a better resolution. According to experience, $c_{s}$ ranges from 0.1 to 1.0. $D_{E}$ is essentially a choice of the density of PTN monitoring points, which will directly affect the resolution of the simulated results. This will be discussed in Section 6.1.

\subsection{Layout Scheme of Monitoring Points}

A large number of monitoring points should be arranged in the simulation area. Different from grid nodes, these monitoring points are used to monitor and record pressure 
pulsation. The layout mode of monitoring points can be adjusted according to the topology of the monitoring area. In the example of a two-dimensional (2D) case, as shown in Figure 1a, the monitoring points can be arranged evenly in the Cartesian coordinate system. For the rotor and stator of rotating machinery, the Cylinder coordinate system could be improved by using the monitoring point arrangement mode shown in Figure $1 \mathrm{~b}$.

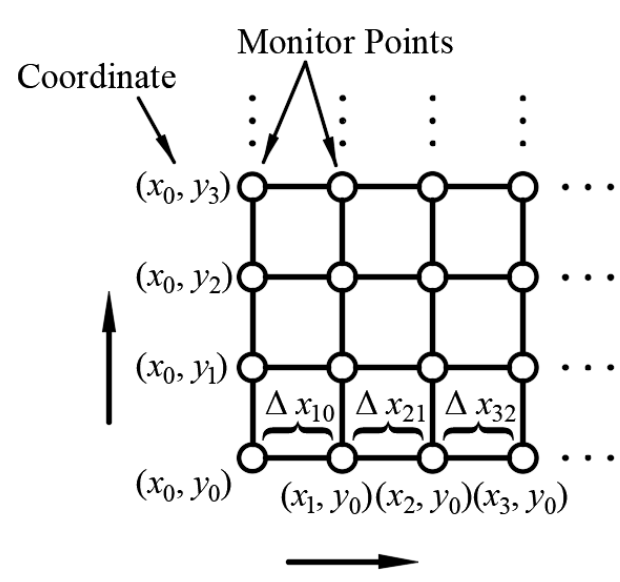

(a)

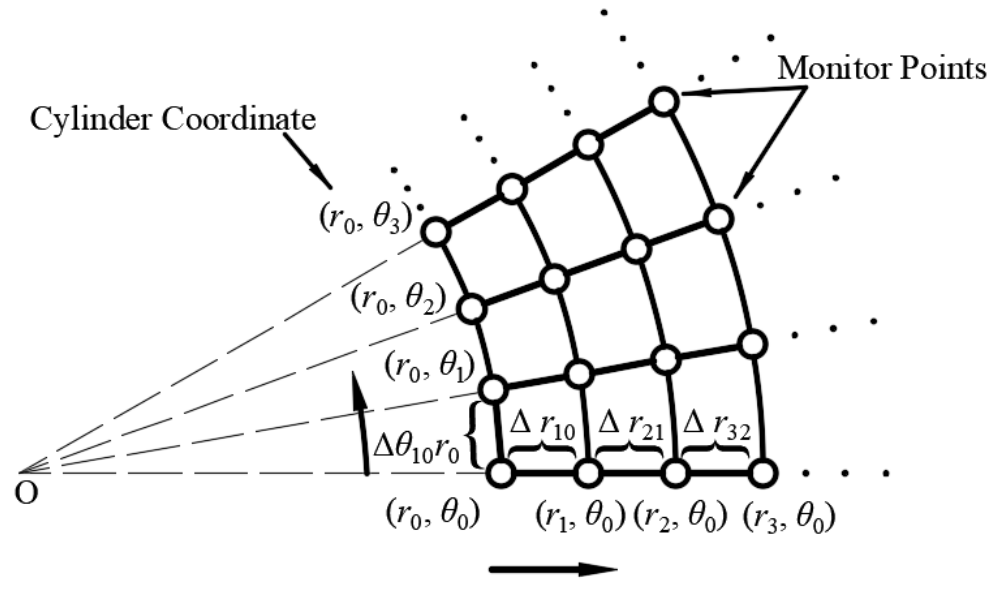

(b)

Figure 1. Layout of monitoring points in the coordinate systems: (a) in the Cartesian system; (b) in the Cylinder system.

\subsection{The Process of Signal Propagation}

A pulsating signal can be propagated in a certain direction in space, and the ideal propagation process is shown in Figure 2. The signal at $P_{1}$ propagates in a certain direction, and it passes one period just after transmitting to $P_{n}$. Each point has phase difference behind the previous point, as shown in Figure 3. However, the amplitude $(A)$ and main frequency $\left(f_{m}\right)$ remain unchanged during propagation.

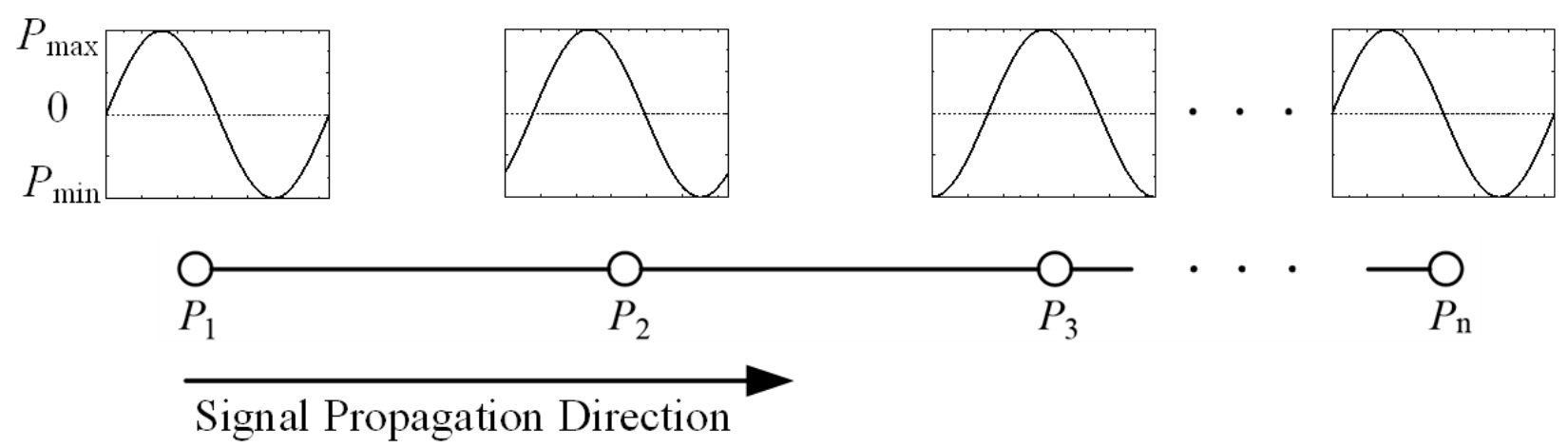

Figure 2. Schematic map of signal propagation.

Because of the signal intensity attenuation, the propagation of $\Delta \varphi$ and $\Delta P$ from $P_{1}$ to $P_{2}$ and from $P_{2}$ to $P_{3}$ was not equal in the actual situation. If another signal comprising other points was propagated here, it would have made the signal more disordered. It was difficult to trace back to the signal source. PTN methods can also provide solutions for signal traceability. 

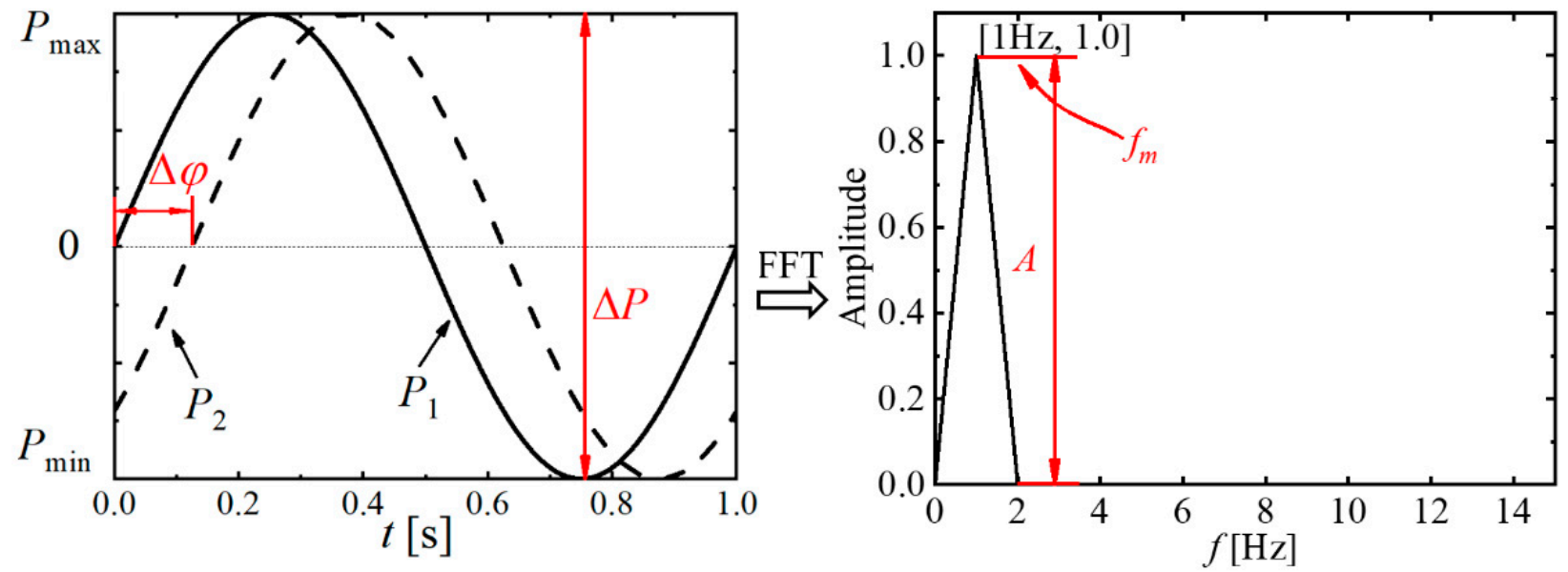

Figure 3. $A$ and $f_{m}$ for the propagation of a signal.

\section{Testing Case Details}

\subsection{Case Description}

As a typical fluid dynamic's phenomenon, trailing-edge vortex shedding generates very regular pulsations. Even though its time-domain and frequency-domain are widely studied by researchers, the relevant pulsation intensity, the cover range of frequencies and the spatial distributions of temporal states are not fully understood. Therefore, this study took the trailing-edge vortex-shedding case of symmetrical NACA0009 foil as the research object $[23,24]$. A comparative case testing process was conducted in this study by comparing traditional analysis and PTN analysis. For simplification in this comparative testing process, the 2D flow domain is discussed as shown in Figure 4. The chord length $L$ was shortened from 110 to $100 \mathrm{~mm}$. The trailing-edge thickness of the hydrofoil was $h_{t}=3.22 \mathrm{~mm}$. The maximum thickness of the hydrofoil was $t_{\max }=9.90 \mathrm{~mm}$. The distance from the maximum thickness to the leading-edge was $L_{m}=49.50 \mathrm{~mm}$. The incidence angle was 0 degrees. The incoming flow velocity $U_{\text {ref }}$ was $20 \mathrm{~m} / \mathrm{s}$ and the Reynolds number $R e_{h}=U_{r e f} h_{t} / v$ was approximately $64.4 \times 10^{3}$. The Strouhal number $S t_{h}=h_{t} f / U_{r e f}$ was approximately 0.19 .
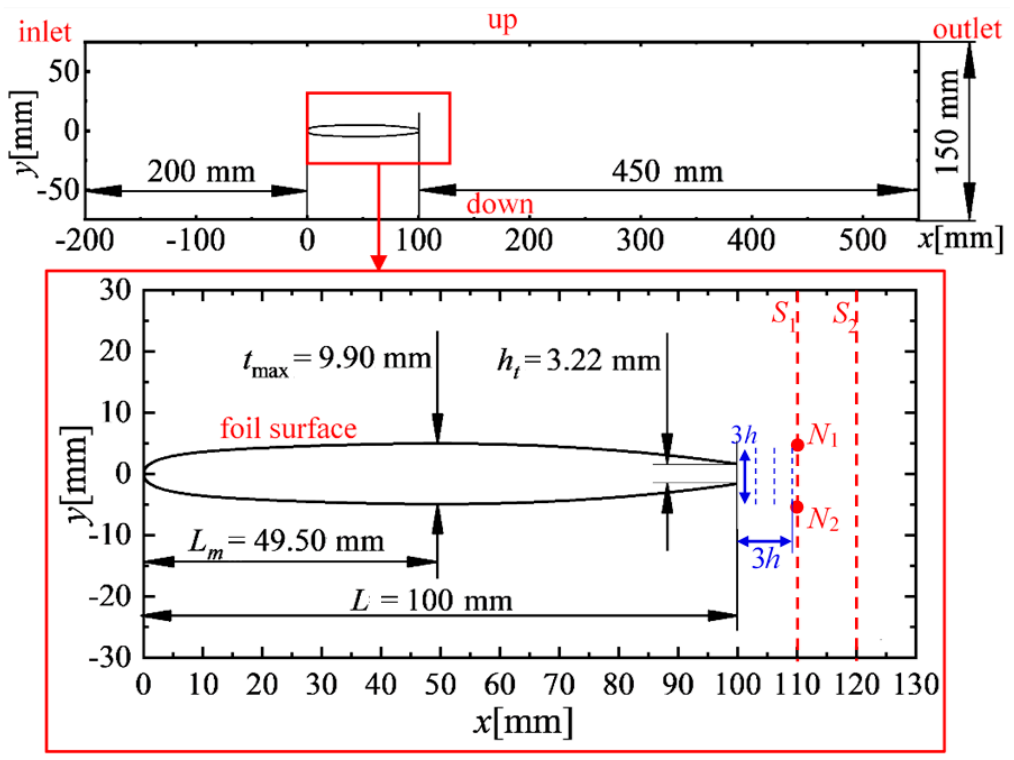

Figure 4. Geometrical diagram of the 2D-CFD case with indications of reference points and lines. 
The ANSYS CFX computational code was utilized to simulate the flow fields. The Reynolds-averaged Navier-Stokes (RANS) equations were used in this case, also taking into account the total energy equation. The SST $k-\omega$ turbulence model [25-27] was used to close the RANS equations. Furthermore, the transport equations of the turbulent kinetic energy $k$ and specific dissipation rate $\omega$ can be expressed as:

$$
\begin{aligned}
\frac{\partial(\rho k)}{\partial t}+\frac{\partial\left(\rho u_{i} k\right)}{\partial x_{i}} & =P-\frac{\rho k^{3 / 2}}{l_{k-\omega}}+\frac{\partial}{\partial x_{i}}\left[\left(\mu+\sigma_{k} \mu_{t}\right) \frac{\partial k}{\partial x_{i}}\right] \\
\frac{\partial(\rho \omega)}{\partial t}+\frac{\partial\left(\rho u_{i} \omega\right)}{\partial x_{i}} & =C_{\omega} P-\beta \rho \omega^{2}+\frac{\partial}{\partial x_{i}}\left[\left(\mu_{l}+\sigma_{\omega} \mu_{t}\right) \frac{\partial \omega}{\partial x_{i}}\right] \\
& +2\left(1-F_{1}\right) \frac{\rho \sigma_{\omega 2}}{\omega} \frac{\partial k}{\partial x_{i}} \frac{\partial \omega}{\partial x_{i}}
\end{aligned}
$$

where $\rho$ is the fluid density, $P$ is the turbulence generation term, $\mu$ is the dynamic viscosity, $\mu_{t}$ is the eddy viscosity coefficient, $\sigma$ is the model constant, $C_{\omega}$ is the coefficient of turbulent dissipation term, $F_{1}$ is the blending equation, and $l_{k-\omega}$ is the scale of turbulence.

The grid scheme used in the 2D computational domain had 81,026 nodes, and the grid elements were quadrilateral structured 2D elements. The range of $y^{+}$on the wall was controlled within the range of $2 \sim 13$ for the application of wall functions in the near wall region. The boundary conditions were set as shown in Table 1. Transient simulation was carried out based on the steady results. The time-step of transient computation was set to $1.0 \times 10^{-5} \mathrm{~s}$. As for the discretization schemes, the central difference scheme was adopted for the diffusion term, the second-order upwind scheme was used for the convective term, while the second-order backward Euler scheme was employed for the transient term. A fully implicit coupling algorithm was employed for transient computation, while the convergence residual standard was set to $1.0 \times 10^{-5}$.

Table 1. Boundary conditions.

\begin{tabular}{cccc}
\hline No. & Position & Type & Description \\
\hline 1 & Inlet & Velocity inlet boundary & $v_{\text {in }}=20 \mathrm{~m} / \mathrm{s}$ \\
2 & Outlet & Pressure outlet boundary & $p_{\text {out }}=1 \mathrm{~atm}$ \\
3 & Foil Surface & Wall & No slip wall \\
4 & Up and Down Walls & Wall & No slip wall \\
5 & Two Sides & Symmetry & Simplified for 2D \\
\hline
\end{tabular}

\subsection{Grid Convergence Check}

To obtain a better reliability for the CFD simulation, the grid convergence index (GCI) was studied based on Richardson's extrapolation method [28]. $G_{1}, G_{2}$ and $G_{3}$ which are three grid schemes, were determined proportionally, as shown in Figure 5. The grid refinement factors were as follows: $r_{21}=1.32$ and $r_{32}=1.31$. The mesh node numbers of the three grids were $\mathrm{G}_{1}=112,844$ ( $x$ : 303 nodes; $y$ : 175 nodes), $\mathrm{G}_{2}$ - 197,240 ( $x$ : 401 nodes; $y$ : 229 nodes) and $\mathrm{G}_{3}=338,200$ ( $x$ : 531 nodes; $y$ : 301 nodes). As indicated in Figure 4, two points $\left(N_{1}(x=110 \mathrm{~m}, y=5 \mathrm{~mm})\right.$ and $\left.N_{2}(x=110 \mathrm{~mm}, y=-5 \mathrm{~mm})\right)$ and two lines $\left(S_{1}(x=110 \mathrm{~mm}), S_{2}(x=120 \mathrm{~mm})\right)$, located near the trailing edge of the hydrofoil, were selected for GCI verification. The parameters $P_{N 1}$ (average value of pressure fluctuation at $N_{1}$ ), $P_{N 2}$ (average value of pressure fluctuation at N2), $P_{S 1}$ (average value of pressure at $\left.S_{1}\right), P_{S 2}$ (average value of pressure at $\left.S_{2}\right), v_{N 1}$ (average value of velocity at $N_{1}$ ) and $v_{N 2}$ (average value of velocity at N2) were used as indexes, and these are listed in Table 2. The apparent-order, represented as $p$, ranged from 1.1813 to 7.2465 , and the extrapolation values $\phi_{\text {ext }}^{21}$ and $\phi_{\text {ext }}^{32}$ were acquired. The approximate relative error $\mathrm{e}_{\mathrm{a}}^{21}$ was in the range of 0.02 to $0.98 \%$, and $\mathrm{e}_{\mathrm{a}}^{32}$ was in the range of 0.09 to $0.40 \%$. The discretization uncertainty $\mathrm{e}_{\mathrm{ext}}^{21}$ was in the range of 0.003 to $0.76 \%$, and $\mathrm{e}_{\mathrm{ext}}^{32}$ was in the range of 0.02 to $1.04 \%$. The convergence index of $\mathrm{G}_{1} \mathrm{GCI}_{\text {fine }}^{21}$ was in the range of 0.004 to $0.94 \%$, and $\mathrm{GCI}_{\text {fine }}^{32}$ was in the range of 0.03 to $1.29 \%$. Hence, $G_{2}$ was selected as a compromise between accuracy and computational cost with a total of 197,240 nodes. 


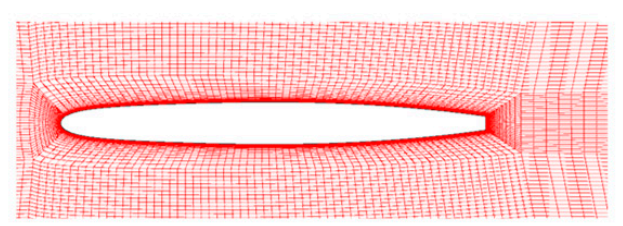

(a)

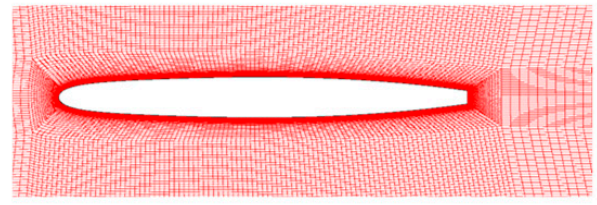

(b)

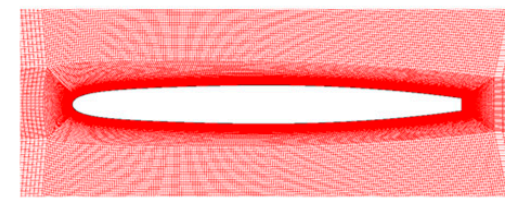

(c)

Figure 5. Three grid schemes: (a) G1, (b) G2, (c) G3.

Table 2. Evaluation results of discretization error in GCI check.

\begin{tabular}{ccccccc}
\hline & $\boldsymbol{P}_{\mathbf{N 1}}(\mathbf{k P a})$ & $\boldsymbol{P}_{\mathbf{N} 2}(\mathbf{k P a})$ & $\boldsymbol{P}_{\mathbf{S} \mathbf{1}}(\mathbf{k P a})$ & $\boldsymbol{P}_{\mathbf{S} 2}(\mathbf{k P a})$ & $\boldsymbol{v}_{\mathbf{N} \mathbf{1}}(\mathbf{m} / \mathbf{s})$ & $\boldsymbol{v}_{\mathbf{N} 2}(\mathbf{m} / \mathbf{s})$ \\
\hline$\phi_{1}$ & 124.71 & 124.65 & 102.58 & 103.85 & 19.256 & 19.248 \\
$\phi_{2}$ & 125.90 & 125.87 & 102.87 & 103.80 & 19.252 & 19.237 \\
$\phi_{3}$ & 126.34 & 126.29 & 103.28 & 103.89 & 19.282 & 19.280 \\
$p$ & 3.9499 & 4.1191 & 1.1813 & 2.3226 & 7.2465 & 4.9176 \\
$\phi_{\text {ext }}^{21}$ & 124.08 & 124.06 & 101.81 & 103.91 & 19.256 & 19.2520 \\
$e_{a}^{21}$ & $0.96 \%$ & $0.98 \%$ & $0.28 \%$ & $0.05 \%$ & $0.02 \%$ & $0.06 \%$ \\
$e_{\text {ext }}^{21}$ & $0.51 \%$ & $0.48 \%$ & $0.76 \%$ & $0.05 \%$ & $0.003 \%$ & $0.02 \%$ \\
$\mathrm{GCI}_{\text {fine }}^{21}$ & $0.63 \%$ & $0.60 \%$ & $0.94 \%$ & $0.07 \%$ & $0.004 \%$ & $0.03 \%$ \\
$\phi_{\text {ext }}^{32}$ & 125.69 & 125.67 & 101.81 & 103.70 & 19.247 & 19.222 \\
$e_{a}^{32}$ & $0.35 \%$ & $0.34 \%$ & $0.40 \%$ & $0.09 \%$ & $0.15 \%$ & $0.22 \%$ \\
$e_{\text {ext }}^{32}$ & $0.17 \%$ & $0.16 \%$ & $1.04 \%$ & $0.10 \%$ & $0.02 \%$ & $0.07 \%$ \\
$\mathrm{GCI}_{\text {fine }}^{32}$ & $0.22 \%$ & $0.20 \%$ & $1.29 \%$ & $0.12 \%$ & $0.03 \%$ & $0.09 \%$ \\
\hline
\end{tabular}

\subsection{Experimental-Computational Validation}

In this study, the velocity of the inlet flow was $20 \mathrm{~m} / \mathrm{s}$. Because of the vortex-shedding, strong pressure and velocity changes downstream of the trailing edge of the hydrofoil were expected. Based on the experimental data [24], the numerical trailing-edge flow field could be validated by considering the decomposition of the velocity with the time-averaged and pulsating components. The two components can be expressed as follows:

$$
\begin{gathered}
U_{\text {mean }}=\frac{1}{N} \sum_{i=1}^{n} U_{i} \\
U_{s t d v}=\sqrt{\frac{1}{N} \sum_{i=1}^{N}\left(U_{i}-U_{\text {mean }}\right)^{2}}
\end{gathered}
$$

where $U_{i}$ is the transient velocity; $U_{\text {mean }}$ is the time-averaged velocity; $U_{\text {stdv }}$ is the root mean square of the velocity component; and $N$ presents the number. The experimentalcomputational validation is shown in Figure 6 by plotting the $U_{\text {mean }} / U_{r e f}$ and $U_{\text {stdv }} / U_{\text {ref }}$ curves on three reference lines. The reference velocity $U_{\text {ref }}$ was equal to the upstream velocity of $20 \mathrm{~m} / \mathrm{s}$. As indicated in Figure 4, the three lines were distributed downstream from the trailing-edge with the distance of $h=h_{t}=3.22 \mathrm{~mm}$ between every two lines. The $y$-direction lengths of the three lines were also $3 h$. For the pulsating velocity in the $x$-direction, $U_{x s t d v}$, the maximum difference was 0.09 between the experimental results and the computational results on the first line, and the maximum difference was 0.10 between the experimental results and the computational results on the third line. It can be found that the time-averaged velocity in the $x$-direction $U_{x \text { mean }}$, the time-averaged velocity in the $y$-direction $U_{y \text { mean }}$, and the pulsating velocity in the $y$-direction $U_{y}$ stdv, were in good agreement with the experimental results. Some researchers $[29,30]$ used the same method to calculate the shedding frequency of the Karman vortex street at the trailing edge of the hydrofoil. 


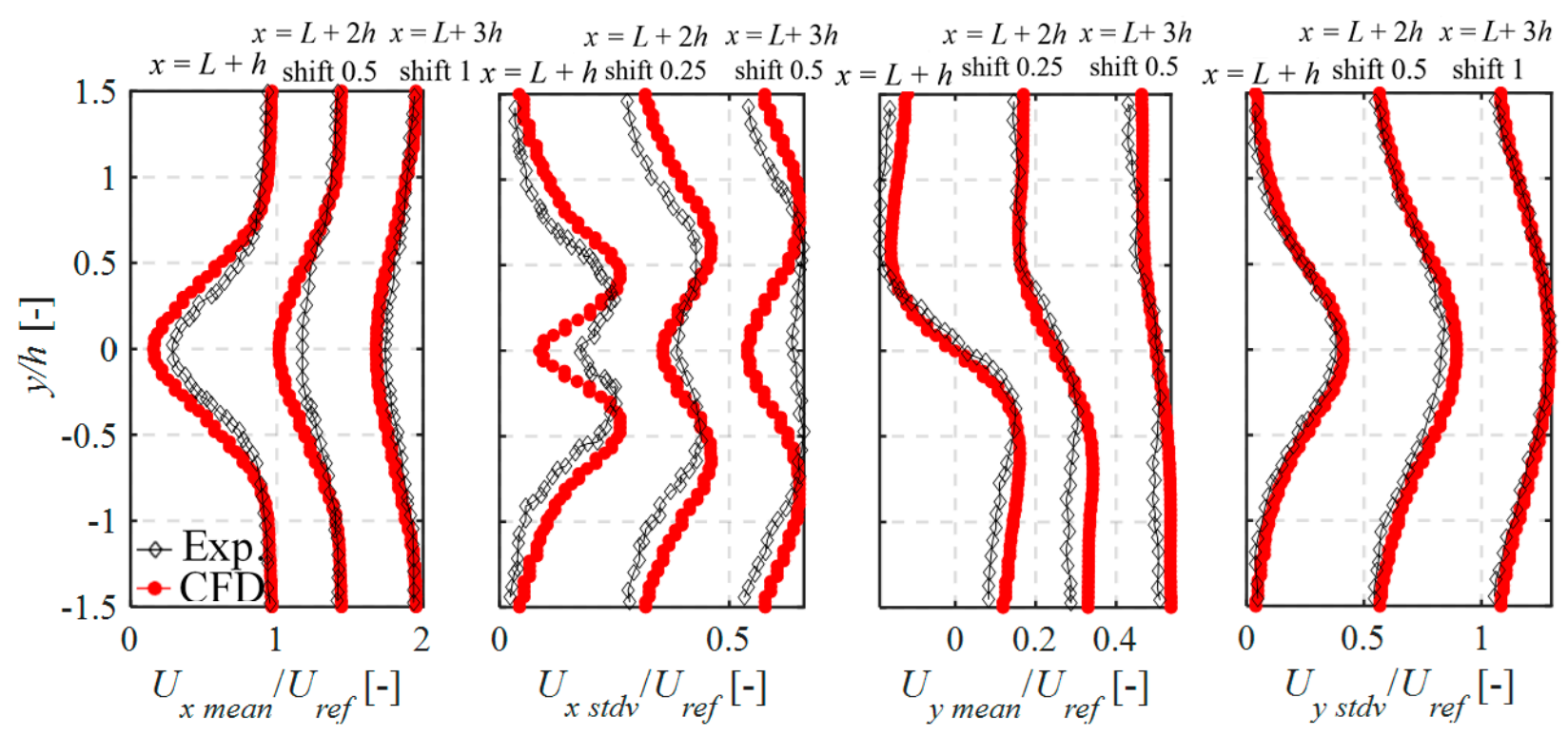

Figure 6. Comparison of experimental and simulated results.

\section{Traditional CFD Post-Processing}

\subsection{Flow Patterns of Trailing-Edge Vortex Shedding}

Based on the CFD simulation result, the flow patterns of trailing-edge vortex shedding can be analysed as shown in Figure 7. Both the pressure $p$ and the dimensionless pressure coefficient $C_{p}$ are tagged as shown in Figure 7a. The dimensionless pressure coefficient $C_{p}$ can be expressed as:

$$
C_{p}=\frac{2\left(p-p_{r e f}\right)}{\rho U_{r e f}^{2}}
$$

where $\rho$ is the density, and $p_{\text {ref }}$ is the reference pressure at the inlet boundary. Local high and low $C_{p}$ sites could be alternately found in the vortex-shedding region. Figure $7 \mathrm{~b}$ shows the contour of velocity. Both the velocity $U$ and normalized velocity coefficient $C_{v}$ are tagged. The velocity coefficient $C_{v}$ can be calculated by:

$$
C_{v}=U / U_{r e f}
$$

Alternatively, local low $C_{v}$ regions could be found fluctuating along the $x$ direction. Both the $C_{p}$ and $C_{v}$ patterns indicated the alternative pattern, which was the typical pattern of the trailing-edge vortex-shedding flow. Therefore, it was necessary to plot the vorticity $\Omega_{z}$ perpendicular to $x-y$ plane in Figure 7c. This clearly shows the shedding vortexes downstream of the trailing edge with the decreasing intensity of $\Omega_{z}$ along the $x$ direction.

\subsection{Interactions among Vortexes}

The decreasing intensity of $\Omega_{z}$ indicates the interactions among the shedding vortexes. Figure 8 a shows the pattern of entropy production and the ISO- $\Omega_{z}$ curves at a specific time step. The entropy production $E_{p}$ can approximately show the energy dissipation due to flow-flow interaction [31]. It can be expressed as:

$$
E_{p}=C_{e} \rho k \varepsilon / T
$$

where $k$ is the kinetic energy of the turbulence, $\varepsilon$ is the eddy dissipation of the turbulence, and $T$ is the temperature. Negative and positive ISO- $\Omega_{z}$ curves represent the instantaneous positions of the vortexes. The high $E_{p}$ sites appeared mainly in the interaction regions between a positive $\Omega_{z}$ vortex and a negative $\Omega_{z}$ vortex. Figure $8 \mathrm{~b}$ shows the pattern of $E_{p}$ and $\Omega_{z}$ of eight continual time steps, with a time difference of $0.0001 \mathrm{~s}$ between each two 
steps. It was found that, in general, the periodic shedding of vortexes would periodically cause high-flow energy dissipation.
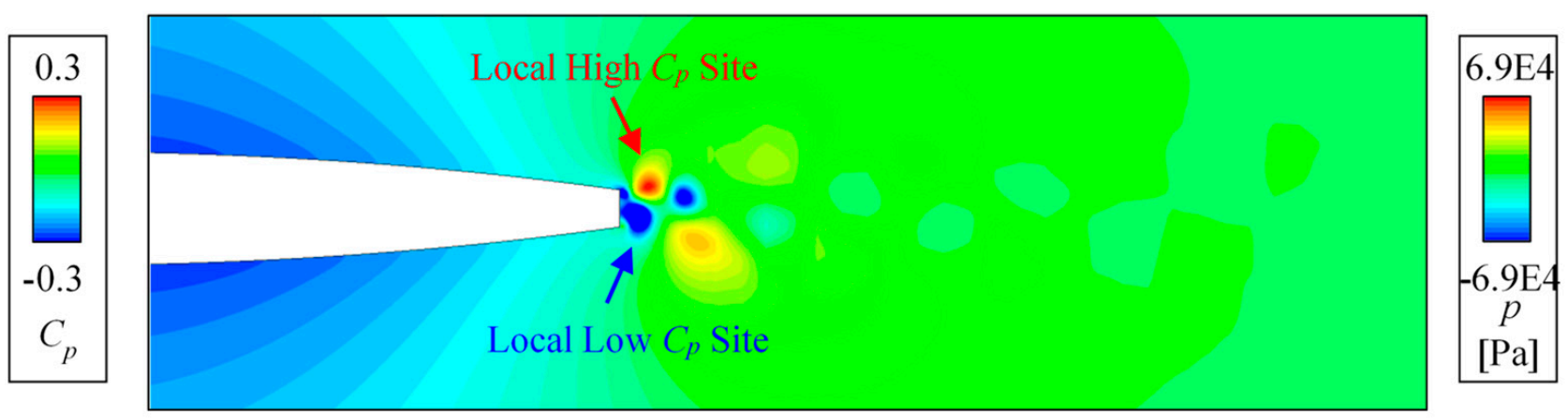

(a)
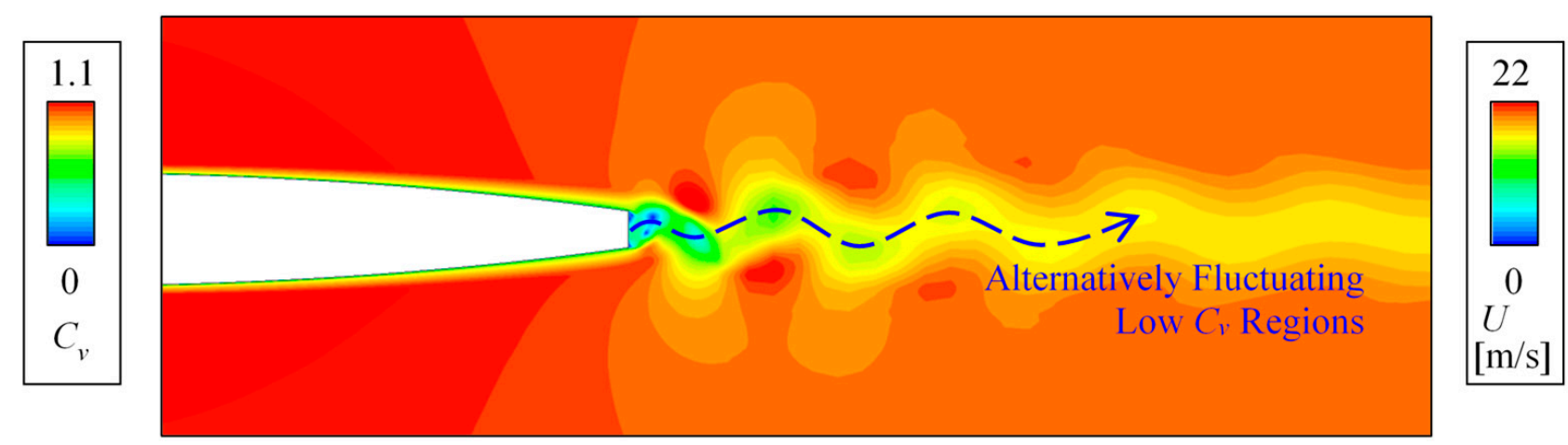

(b)

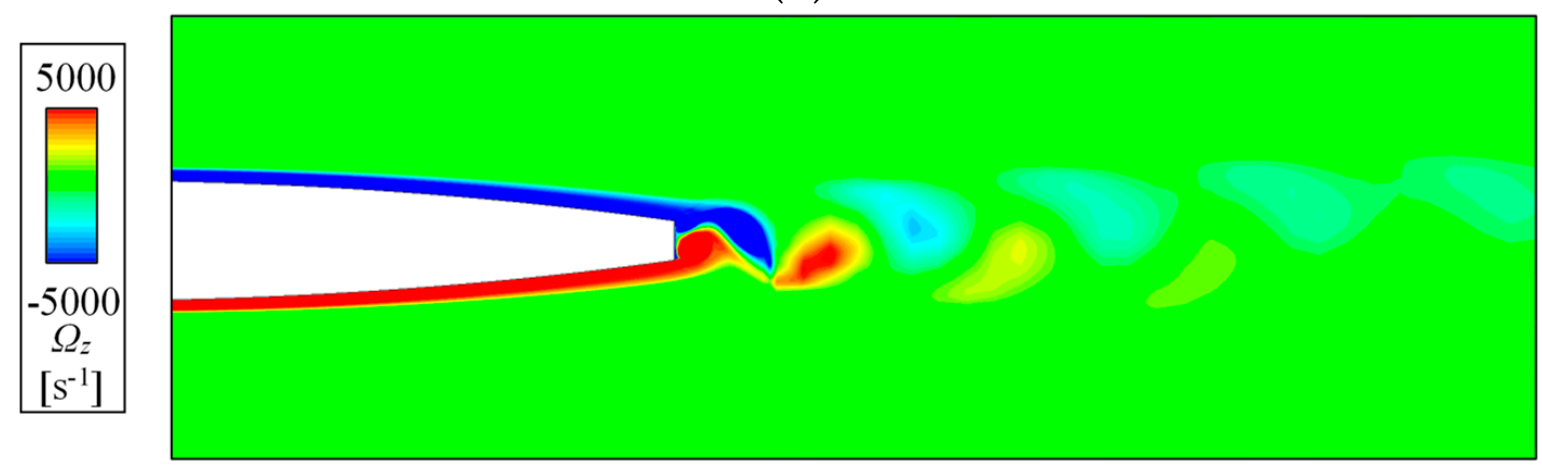

(c)

Figure 7. Flow patterns of trailing-edge vortex shedding of hydrofoil. (a) Pressure coefficient $C_{p}$. (b) Velocity coefficient $C_{v}$. (c) Vorticity perpendicular to the $x-y$ plane of $\Omega_{z}$.

\subsection{Pressure Pulsation on Typical Sites}

Pressure pulsation is one of the most important transient phenomena in vortexshedding flow cases. Therefore, in this study, it was necessary to analyse the pressure pulsation with both time-domain and frequency-domain plots. In traditional pressure pulsation analyses, discrete monitoring points can be distributed in the concerned region. As shown in Figure 9a, a total of nine points were set in the vortex-shedding region, and were denoted as $T_{1} \sim T_{3}, M_{1} \sim M_{3}$ and $D_{1} \sim D_{3}$. The distance between two adjacent points was $5 \mathrm{~mm}$ and the distance between $\mathrm{M}_{1}$ and the trailing-edge was also $5 \mathrm{~mm}$. 


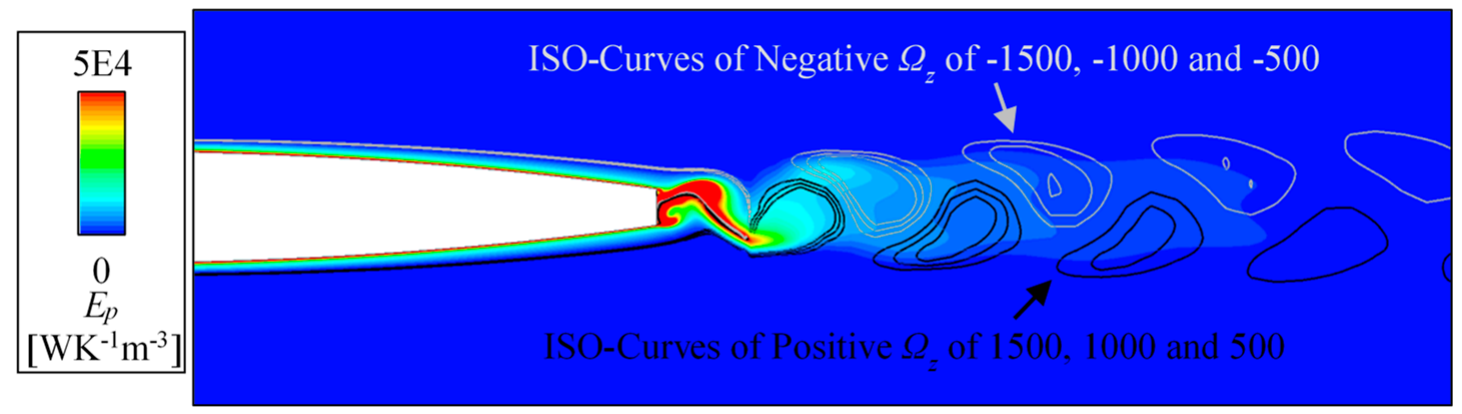

(a)
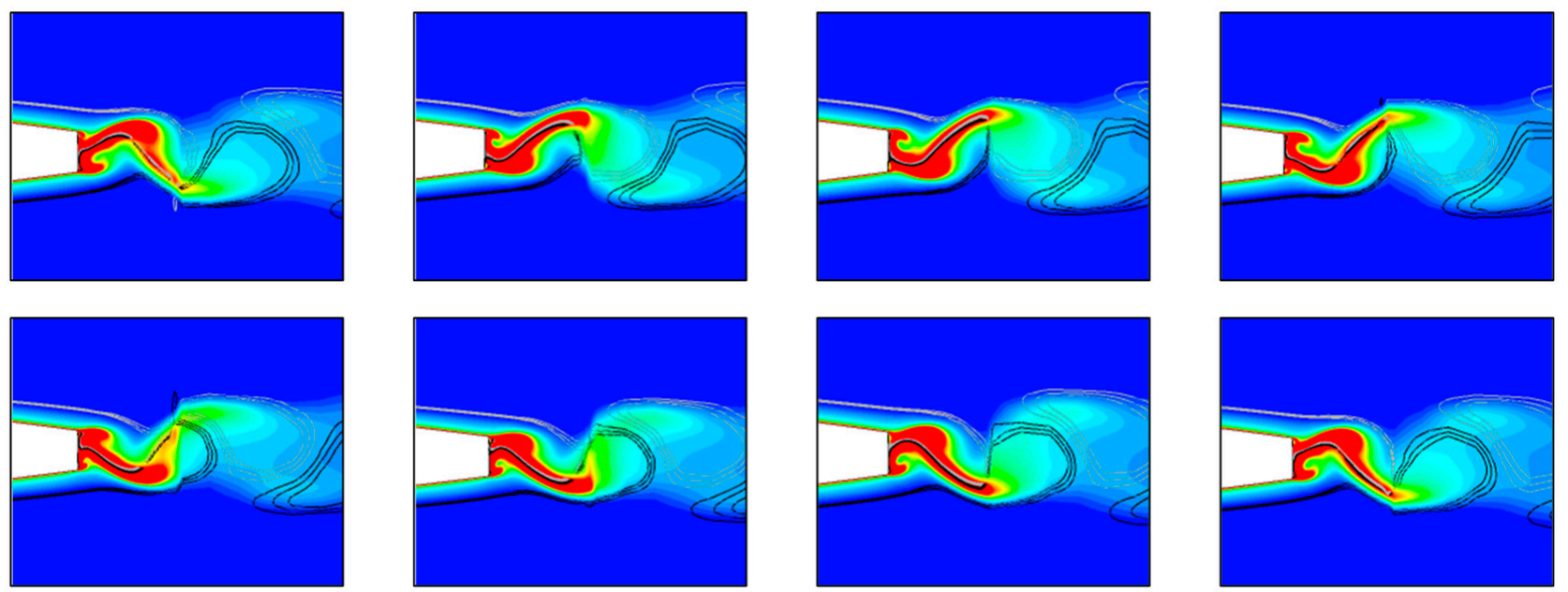

(b)

Figure 8. Temporal variation of vortex shedding with interactions. (a) Pattern of entropy production and $\Omega_{z}$ at a specific time step. (b) Pattern of 8 continual time steps with $0.0001 \mathrm{~s}$ between every two steps.

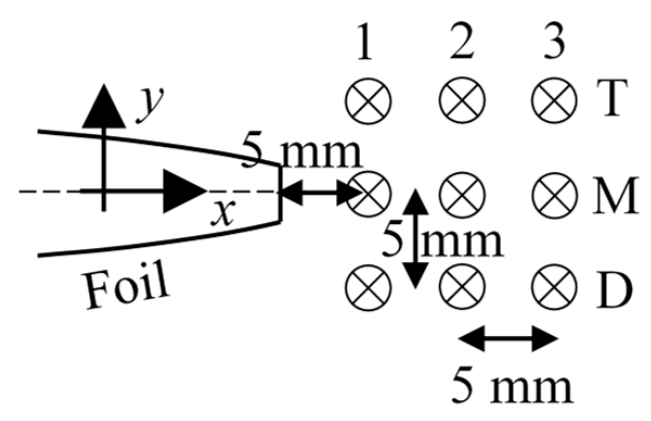

(a)

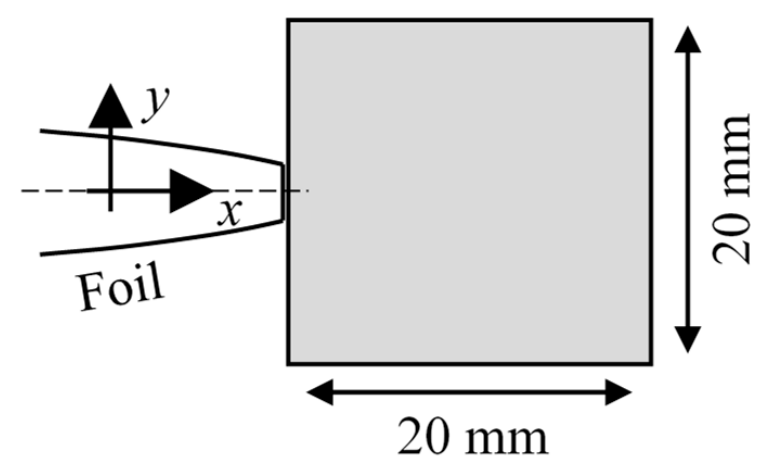

(b)

Figure 9. Monitoring points for pressure pulsation analysis. (a) Discrete monitoring points for traditional pressure pulsation analysis. (b) PTN monitoring for a specific region.

Figure 10 includes the time-domain plot and frequency-domain plot of the pressure pulsation on the nine points. The time-domain waveforms were strongly periodic within $0.006 \mathrm{~s}$. The pressure values on $\mathrm{T}_{1} \sim \mathrm{T}_{3}$ and $\mathrm{D}_{1} \sim \mathrm{D}_{3}$ were similar, which shows the symmetry of vortex-shedding flow. The pressure values on $\mathrm{M}_{1} \sim \mathrm{M}_{3}$ were around 0 Pa because $\mathrm{M}_{1} \sim \mathrm{M}_{3}$ were approximately located on the $y=0$ line. On the frequency-domain plot of the root mean square (RMS) value of pressure, two special frequencies were found. The $1152 \mathrm{~Hz}$ frequency denoted by $f_{v s}$ was the main frequency of points $\mathrm{T}_{1} \sim \mathrm{T}_{3}$ and $\mathrm{D}_{1} \sim \mathrm{D}_{3}$. The $2304 \mathrm{~Hz}$ 
frequency denoted by $2 f_{v s}(1152 \mathrm{~Hz} \times 2)$ was the main frequency of points $\mathrm{M}_{1} \sim \mathrm{M}_{3}$. It was clear that $f_{v s}$ was the vortex-shedding frequency. $\mathrm{T}_{1} \sim \mathrm{T}_{3}$ and $\mathrm{D}_{1} \sim \mathrm{D}_{3}$ were dominated by this frequency because they were symmetrically distributed on the two sides of $y=0$ line. $\mathrm{M}_{1} \sim \mathrm{M}_{3}$ were dominated by $2 f_{v s}$ because they were located in the interaction region between two vortex rows. However, the influence range and variation law of $f_{v s}$ and $2 f_{v s}$ could not be fully understood based on the traditional method of analysis. Phase and phase differences could be also found on the time-domain plot but were not very clear for the purpose of comparison and analysis.

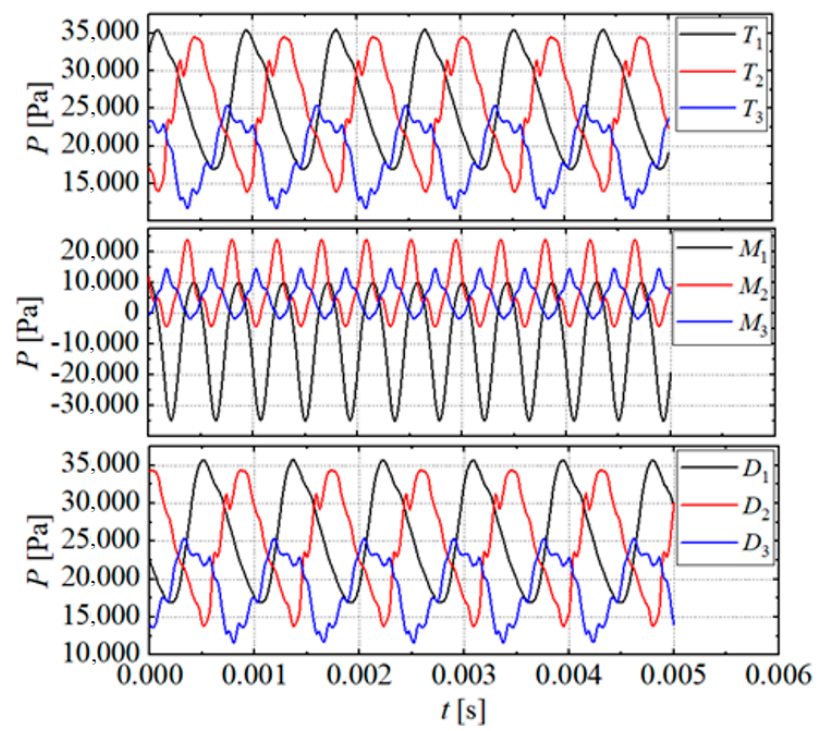

(a)

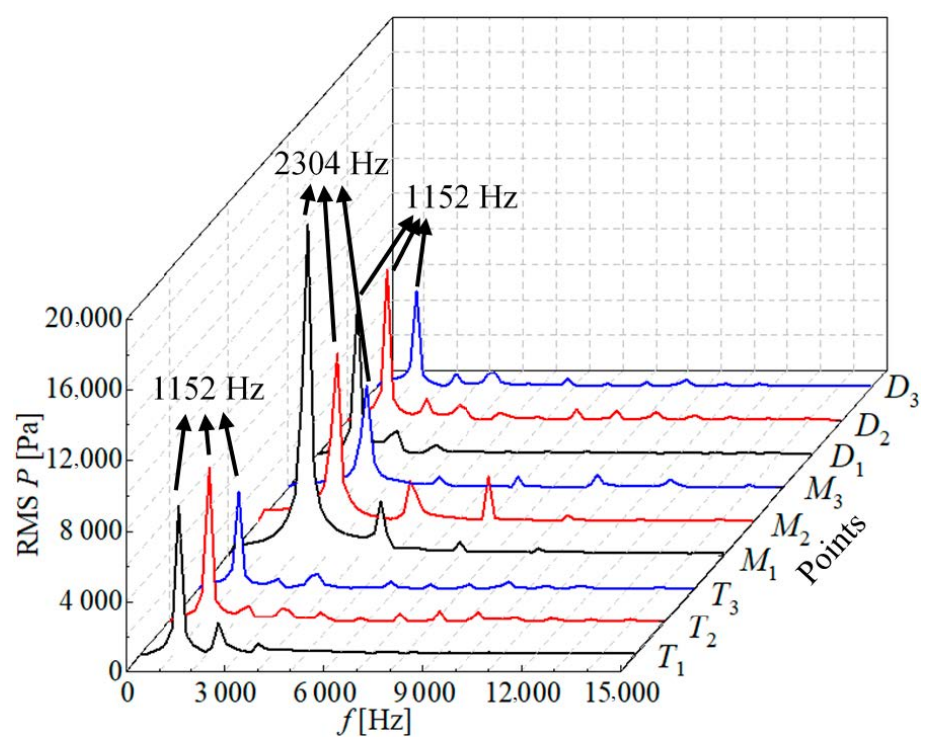

(b)

Figure 10. The pressure pulsations on monitoring points $T_{1} \sim T_{3}, M_{1} \sim M_{3}$ and $D_{1} \sim D_{3}$. (a) Time-domain plot. (b) Frequencydomain plot.

\section{PTN Tracking Results}

\subsection{Setup of PTN}

In Section 3.1, the parameter $c_{S}$ was defined to adjust the density of the layout of the monitoring points. The graphic resolution of different $c_{S}$ values is discussed in this part. The specific selected parameters are analysed in detail in the following sections, as shown in Figure 11, which includes a comparison of $c_{S}=0.145,0.290,0.435,0.580,0.725$ and 1.000. It can be seen that the graphic resolution directly affected the display result. Low resolution led to the over-enlargement of high and low areas, and the capture of spatial distribution was not sufficiently smooth. In this study, the result with the smallest $c_{S}$ was used for analysis in order to obtain high-resolution simulation results.

As shown in Figure 9b, a total of 10,201 monitoring points were distributed in an evenly-spaced pattern downstream of the trailing edge of the hydrofoil. These points were coded by X000 X100 along the $x$-direction and Y000 Y100 along the $y$-direction. The parameters in Equation (2) could thus be determined as $D_{E}=0.2 \mathrm{~mm}$ and $c_{S}=0.145$. Therefore, these 10,201 points covered a $20 \mathrm{~mm} \times 20 \mathrm{~mm}$ region. The relative point position $X$ and $Y$ are used in the following sections for a better comparative analysis. In the following PTN analyses, the total sampling time is $0.1 \mathrm{~s}$.

\subsection{Amplitude}

As found in Figure 10, the vortex-shedding frequencies $f_{v s}$ and $2 f_{v s}$ were the dominant frequencies in this case. Thus, the RMS amplitude values of $f_{v s}$ and $2 f_{v s}$ were analysed based on the PTN, as shown in Figure 12. As shown in Figure 12a, the RMS amplitude of $f_{v s}$ was symmetrically distributed (along $Y=50$ ) downstream of the trailing edge of the hydrofoil because the vortex was alternately shedding in two rows. The strongest 
$f_{v s}$ regions were located at approximately $X=6, Y=54$ and $X=6, Y=46$. The highest amplitude of $f_{v s}$ was approximately $75,000 \mathrm{~Pa}$. Then, the intensity of $f_{v s}$ decreased, mainly along two directions, as indicated. In the region between the two strongest regions, which was also along the $Y=50$ line, the intensity of $f_{v s}$ was very low. However, this was a strong region of $2 f_{v s}$ amplitude. The strongest $2 f_{v s}$ region was at approximately $X=10, Y=48 \sim 50$, which was almost-but not perfectly—symmetrical because of the limited sampling time. The strong $2 f_{v s}$ region distribution also fluctuated along the $x$-direction due to the vortex street. The highest amplitude of $2 f_{v s}$ was approximately $7100 \mathrm{~Pa}$, which was only $1 / 10$ of $f_{v s}$. The range from $X=30$ to $X=90$ constituted a region where $2 f_{v s}$ was visible but $f_{v s}$ was very weak. Generally, both $f_{v s}$ and $2 f_{v s}$ attenuated in the far-away field from the $Y=50$ line, and this attenuation downstream.

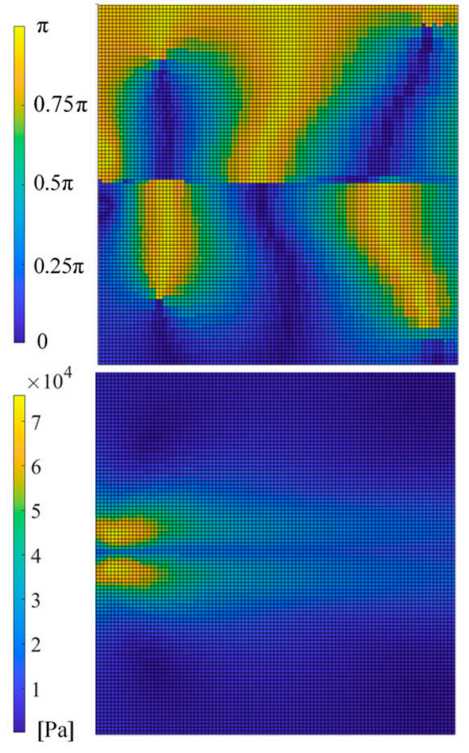

(a)

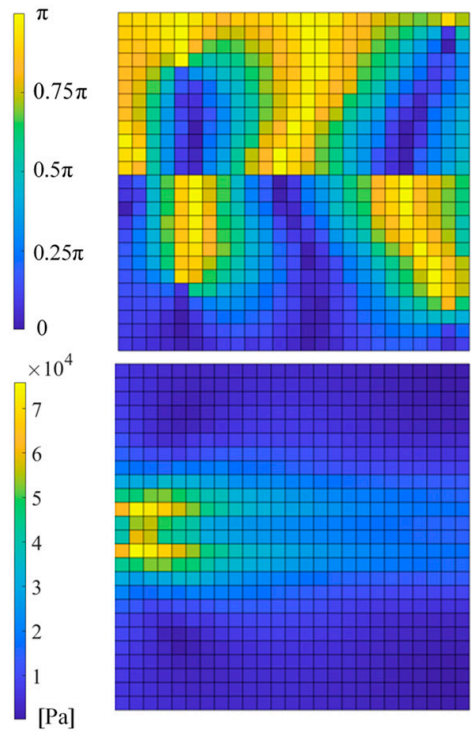

(d)

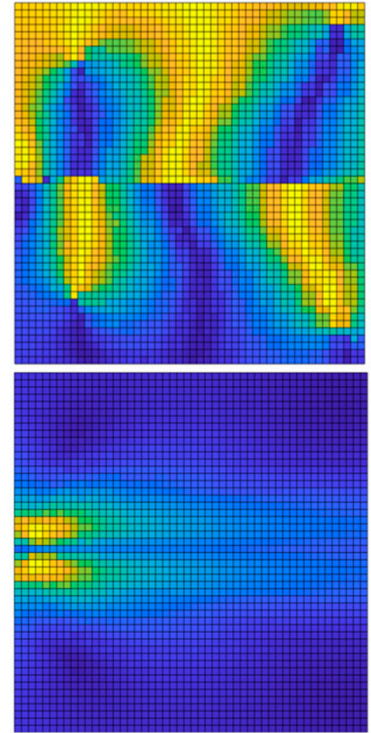

(b)
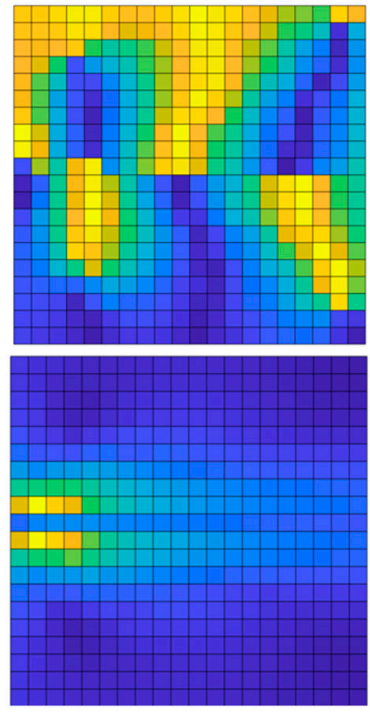

(e)
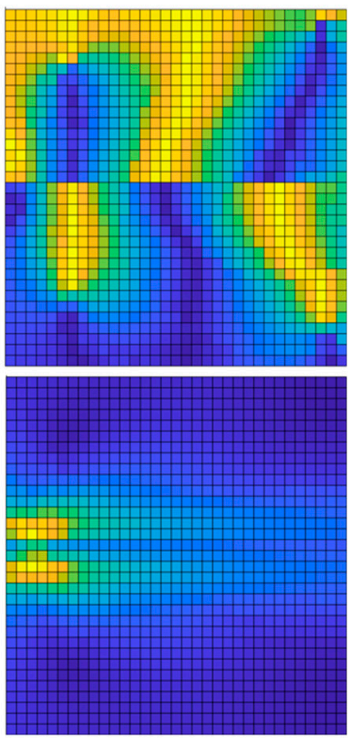

(c)
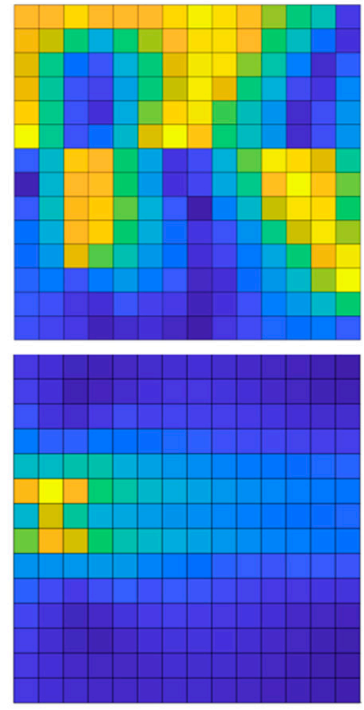

(f)

Figure 11. The graphics resolution of different $c_{S}$ values. $(\mathbf{a}) c_{S}=0.145$ (number of points: $101 \times 101$ ). (b) $c_{S}=0.290$ (number of points: $51 \times 51$ ). $(\mathbf{c}) c_{S}=0.435$ (number of points: $34 \times 34$ ). $(\mathbf{d}) c_{S}=0.580$ (number of points: $26 \times 26$ ). $(\mathbf{e}) c_{S}=0.725$ (number of points: $21 \times 21$ ). (f) $c_{S}=1.000$ (number of points: $15 \times 15$ ). 


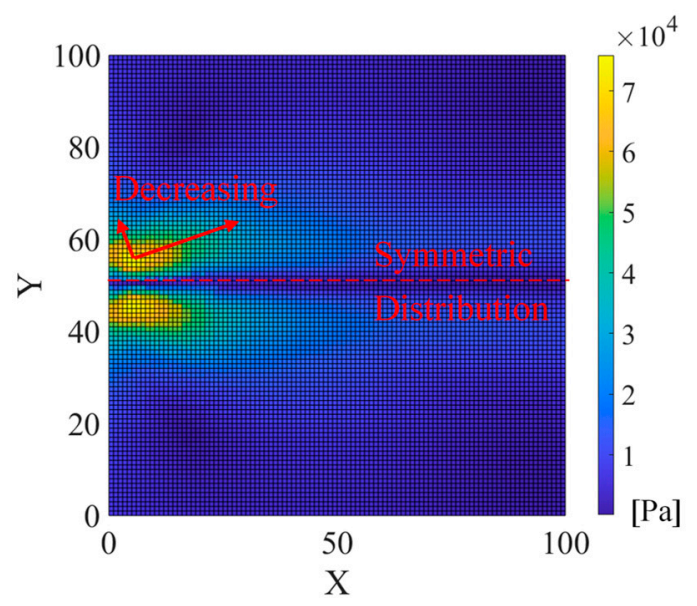

(a)

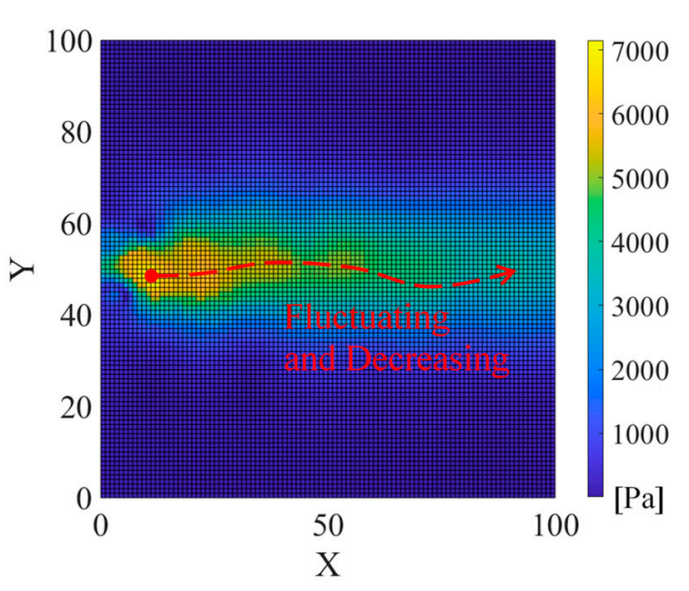

(b)

Figure 12. The distribution characteristics of the pulsation amplitude. (a) RMS amplitude distribution of $f_{v s}$. (b) RMS amplitude distribution of $2 f_{v s}$.

\subsection{Intensity and Attenuation}

Figure 13 shows the plot of the pressure pulsation peak-to-peak values $\Delta P$ (at $97 \%$ confidence intervals). In this study, it was used to monitor the spatial attenuation of the total pressure pulsation intensity including all of the frequency components. The pattern of $\Delta P$ was strongly similar to the pattern of $f_{v s}$ amplitude. It was also symmetrically distributed (along $Y=50$ ) under the influence of two-rows of local shedding vortex flow. This indicates that $f_{v s}$ was, without a doubt, the most dominant frequency in the monitored region. However, there was also a difference between the $\Delta P$ pattern and the $f_{v s}$ amplitude pattern in that the intensity of $\Delta P$ was not so weak in the $X=30 \sim X=90$ region on the $Y=50$ line. This was because of the existence of local high intensity component of $2 f_{v s}$.

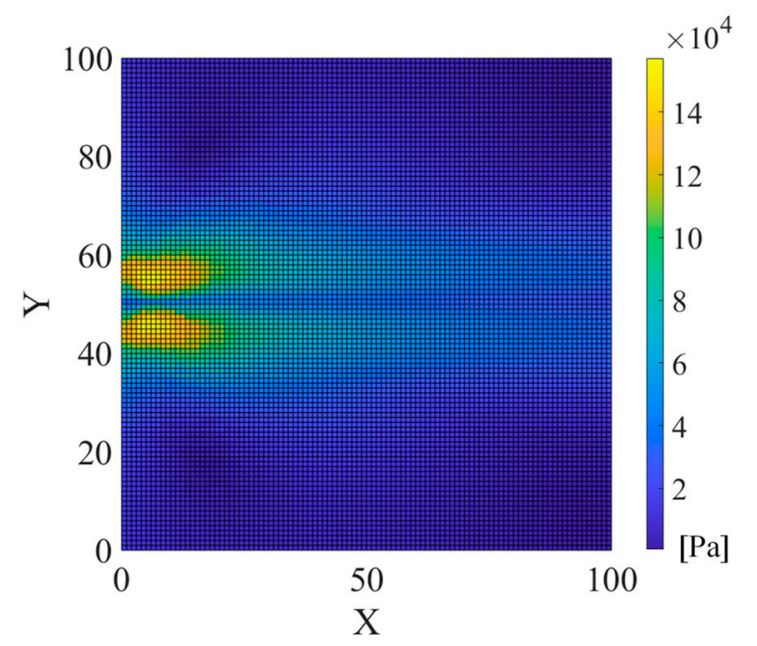

Figure 13. The distribution of pressure pulsation peak-to-peak value $\Delta P$.

\subsection{Dominant Frequency Distribution}

The dominant frequency distribution, which is shown in Figure 14, was obtained by analysing the dominant frequency at every PTN point. The results show that there were only two dominant frequencies, which were $1152 \mathrm{~Hz}$ and $2304 \mathrm{~Hz}$. As described above, the $1152 \mathrm{~Hz}$ was $f_{v s}$ and is shown in blue. The $2304 \mathrm{~Hz}$ was $2 f_{v s}$ and is shown in yellow. It was found that the $2 f_{v s}$ frequency distribution was symmetrically diffused in a "jet-shape". In detail, the $2 f_{v s}$ yellow region was very narrow (approximately $\Delta Y=2$ ) on the trailing edge of the foil because the vortex shedding occurred locally on a one-by-one basis. However, it became increasingly wide along the flow direction due to the interaction between the 
two rows of vortexes. At $X=100$, the width of $2 f_{v s}$ yellow region was approximately $\Delta Y=13$. The diffusing ratio was initially quick but became slow after $X>50$. Based on the interaction among the vortexes, as shown in Figure 7 , the energy of the vortexes was also found to attenuate along the $x$-direction. for this reason, $2 f_{v s}$ became stronger, $f_{v s}$ became weaker, and both $f_{v s}$ and $2 f_{v s}$ were continually attenuating.

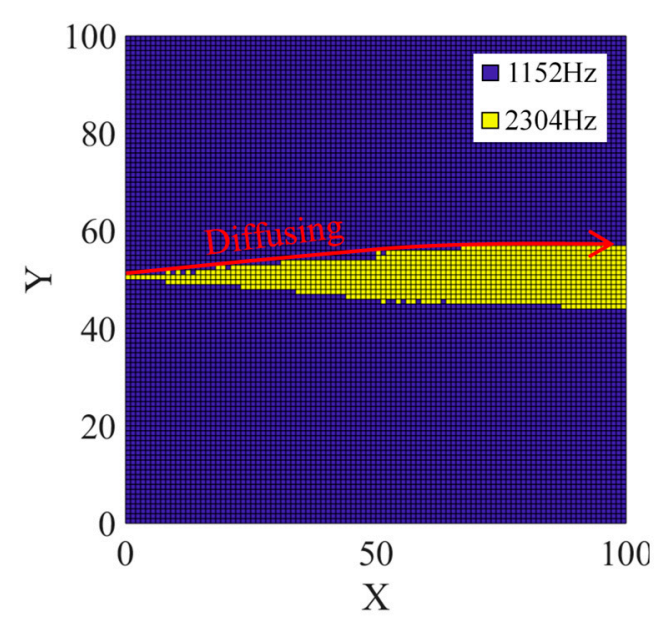

Figure 14. The distribution of dominant frequencies $f_{v s}$ and $2 f_{v s}$.

\subsection{Phase and Phase Difference}

The analysis of the phase and phase difference, based on PTN, demonstrated in this study represents breakthrough in terms of the understanding of pressure pulsation, as compared to traditional analysis. Firstly, it was necessary to set an initial reference point. In this case, the initial reference point $P_{\text {ini }}$ was located on the centre of the trailing edge of the hydrofoil $(X=0, Y=50)$, as illustrated. The phase of $f_{v s}$ on the initial point was defined as the initial phase. The parameter $\Delta \varphi$ represents the phase difference between any point and an initial point, and this is plotted in Figure 15a.

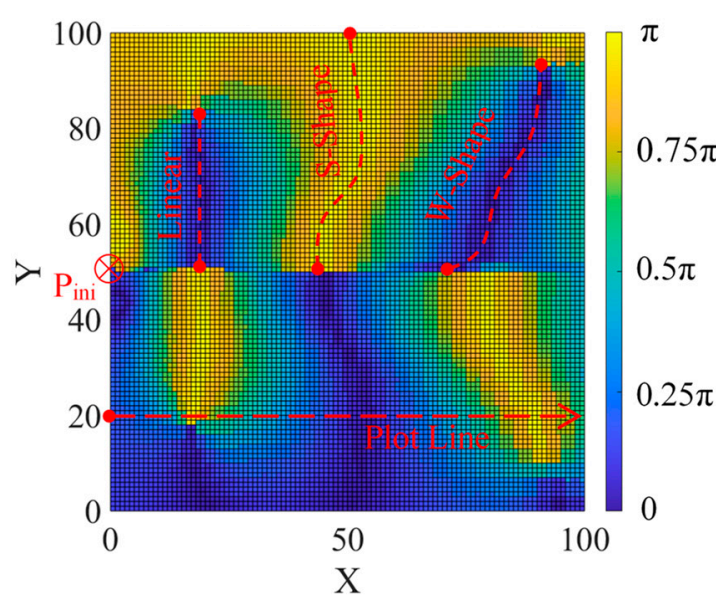

(a)

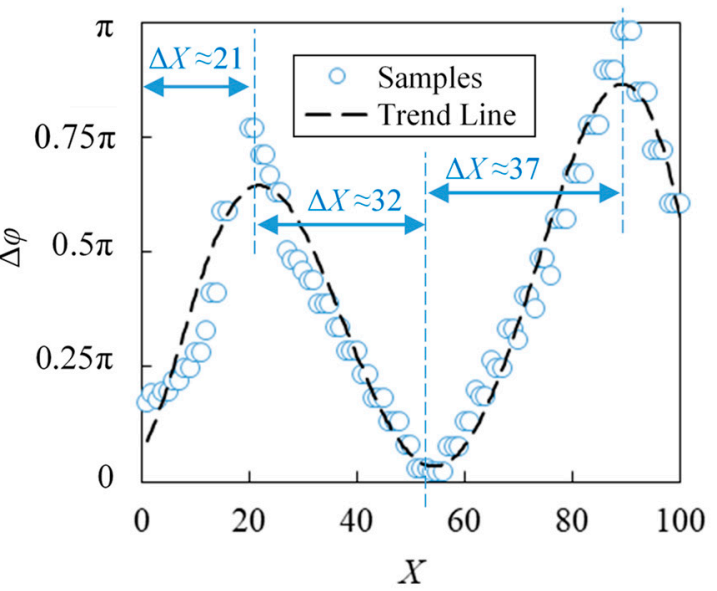

(b)

Figure 15. Distribution of phase difference $\Delta \varphi$. (a) Contour of $\Delta \varphi$. (b) Distribution curve on $Y=20$ plot line.

The above Figure shows an antiphase-symmetrical distribution from the blue-coloured to the yellow-coloured areas where the phase difference $\Delta \varphi$ increased from 0 to $\pi$. in terms of the region between the $Y=50$ line and $Y=80$ line, as an example, it can be seen that $\Delta \varphi$ decreased to 0 at approximately $X=20$. The local low $\Delta \varphi$ area $(\Delta \varphi \approx 0)$ was distributed linearly as illustrated by red dash line. Then, $\Delta \varphi$ increased from 0 to $\pi$. The $X$ position, where $\Delta \varphi=\pi$ on $Y=50$ line, was approximately $X=46$. However, $\Delta \varphi=\pi$ was not linear, 
but had a curvilinear "S-shape", as also illustrated. Then, $\Delta \varphi$ decreased again to 0 . The $\Delta \varphi=0$ position on $Y=50$ line was approximately $X=73$. The local $\Delta \varphi=0$ position became a more complex curve like "W-shape". The transition from linear-shape to "S-shape" to "W-shape" shows that the flow regime became increasingly complex.

Taking $Y=20$ line as another example, the distribution of $\Delta \varphi$ is plotted in Figure 15b. Fitted by trend line, the $X-\Delta \varphi$ relationship can be written as a polynomial formulation:

$$
\Delta \varphi=\sum_{i=0}^{6} C_{x i} X^{i}
$$

where $C_{x i}$ is the coefficient of the $i$-th order term. The values of $C_{x i}$ are listed in Table 3 . The $\mathrm{R}^{2}$ value of this fitting is approximately 0.913 , which indicates goodness of fit. If $\Delta \varphi=0$ is defined as a valley and $\Delta \varphi=\pi$ is defined as a peak, the peak-valley distances along the $x$-direction are wider and wider. As shown, the $\Delta X$ values on the $Y=20$ plot line between the peaks and valleys were approximately 21,32 and 37 within $X=0 \sim 100$. However, the increasing rate of $\Delta X$ became slower with the increasing of $X$. It is worth noting that this phenomenon may indicate the flow-wave attenuation.

Table 3. The values of $C_{x i}$ of the polynomial $X-\Delta \varphi$ trend line.

\begin{tabular}{ccc}
\hline Coefficient & Order of Polynomial Term & Value \\
\hline$C_{x 0}$ & 0 & $2.214 \times 10^{-1}$ \\
$C_{x 1}$ & 1 & $4.896 \times 10^{-2}$ \\
$C_{x 2}$ & 2 & $1.129 \times 10^{-2}$ \\
$C_{x 3}$ & 3 & $-7.031 \times 10^{-4}$ \\
$C_{x 4}$ & 4 & $1.412 \times 10^{-5}$ \\
$C_{x 5}$ & 5 & $-1.165 \times 10^{-7}$ \\
$C_{x 6}$ & 6 & $3.391 \times 10^{-10}$ \\
\hline
\end{tabular}

\section{Conclusions and Discussions}

Based on CFD simulation and an experimental flow validation process, the pressure pulsation characteristics of tracking and processing in a hydrofoil trailing-edge vortexshedding case, were shown. The following conclusions can be drawn:

(1) CFD simulation with a proper time-frequency conversion algorithm, was shown to be effective in predicting the pulsation of turbulent flow fields. By setting discrete monitoring points on important sites, the dominant frequencies in a pulsation signal could be found. However, the traditional time-domain plot and frequency-domain plot were shown to be insufficient in terms of understanding the detailed influences of different pulsation frequencies. As an effective supplement, the pulsation tracking network (PTN) was helpful in resolving pulsating turbulent flow fields. The total pulsation intensity, dominant frequency, amplitude of frequencies, phase and phase difference could be well understood. The influence range, attenuation law and propagation law could be tracked. PTN was found to be very useful as a breakthrough in CFD post-processing.

(2) In this study with a hydrofoil trailing-edge vortex-shedding flow case, PTN was proven to be effective in detailed analyses. Under the Reynolds number of $2.2 \times 10^{6}$, the dominant frequencies were found to be $f_{v s}=1152 \mathrm{~Hz}$ and $2 f_{v s}=2304 \mathrm{~Hz}$. The highest amplitude regions of $f_{v s}$ were symmetrically distributed because of the two shedding vortex rows. The highest amplitude region of $2 f_{v s}$ was slightly downstream to the trailing edge. It was caused by the interaction among vortexes. The $f_{v s}$ frequency was dominant in most of the PTN monitored region. However, the $f_{v s}$ intensity continually attenuated along $x$-direction with an increasingly wider $2 f_{v s}$ dominant region. The $2 f_{v s}$ frequency also attenuated along a fluctuating $x$-direction route. The phase difference contour and curves adequately proved the attenuation. The distances 
between peaks and valleys continually widened along the $x$-direction but this rate of increasing became slower over time.

Discussions are given as follows. In general, PTN was found to be much better than the traditional method in terms of analysing pulsating flow fields. Firstly, it accurately showed the sites with the strongest pulsation intensities, which may be missed in traditional analyses. For example, the maximum value of the RMS amplitude of $f_{v s}$ was found to be less than 20,000 $\mathrm{Pa}$ according to the traditional method, but was actually found to be over 70,000 Pa when using PTN tracking. Secondly, unknown frequencies could be tracked by understanding their source, reason and attenuation. For example, in traditional analysis, $f_{v s}$ and $2 f_{v s}$ can be only speculated upon as the relevant frequencies for vortex-shedding. By using PTN, the regions dominated by $f_{v s}$ and $2 f_{v s}$ were found to overlap the positions of the shedding vortexes. The source, reason and attenuation of $f_{v s}$ and $2 f_{v s}$ thus became much clearer. The spatial distribution of temporal states could be adequately shown. Thirdly, in future applications, the phase distribution will be useful in terms of solving actual engineering flow problems. The route in which the phase varies from 0 to $\pi$ to 0 will indicate hidden directions of flow wave propagation.

Author Contributions: Funding acquisition, R.T.; Methodology, F.J.; Project administration, R.T.; Supervision, R.X.; Validation, R.X.; Visualization, Z.L.; Writing-original draft, F.J.; Writing-review and editing, F.J. All authors have read and agreed to the published version of the manuscript.

Funding: The authors would like to acknowledge the financial support of the National Natural Science Foundation of China. This study was funded by the National Natural Science Foundation of China under grant number 51909131, and the National Natural Science Foundation of China under grant number 51879265 .

Institutional Review Board Statement: Not applicable.

Informed Consent Statement: Not applicable.

Data Availability Statement: Not applicable.

Conflicts of Interest: The authors declare no conflict of interest.

\section{References}

1. Mittal, S. Computation of three-dimensional flows past circular cylinder of low aspect ratio. Phys. Fluids 2001, 13, 177-191. [CrossRef]

2. Voorhees, A.; Dong, P.; Atsavapranee, P.; Benaroya, H.; Wei, T. Beating of a Circular Cylinder Mounted as an Inverted Pendulum. J. Fluid Mech. 2008, 610, 217-247. [CrossRef]

3. Wei, Z.; Zang, B.; New, T.H.; Cui, Y. A proper orthogonal decomposition study on the unsteady flow behaviour of a hydrofoil with leading-edge tubercles. Ocean Eng. 2016, 121, 356-368. [CrossRef]

4. Towne, A.; Schmidt, O.T.; Colonius, T. Spectral proper orthogonal decomposition and its relationship to dynamic mode decomposition and resolvent analysis. J. Fluid Mech. 2018, 847, 821-867. [CrossRef]

5. Hall, K.C.; Thomas, J.P.; Dowell, E.H. Proper Orthogonal Decomposition Technique for Transonic Unsteady Aerodynamic Plows. AIAA J. 2000, 38, 1853-1862. [CrossRef]

6. Lumley, J.L. The structure of inhomogeneous turbulent flows. In Atmospheric Turbulence and Radio Wave Propagation; Nauka: Moscow, Russia, 1967; pp. 166-178.

7. Hussain, A.K.M.F. Coherent structures in turbulence. J. Fluid Mech. 1986, 173, 303-356. [CrossRef]

8. Hutchinson. Stochastic Tools in Turbulence; IOP Publishing Ltd.: Bristol, UK, 2007; Volume 22, p. 161.

9. Sirovich, L. Turbulence and the Dynamics of Coherent Structures. I-Coherent Structures. II-Symmetries and Transformations. III-Dynamics and Scaling. Q. Appl. Math. 1987, 45, 561-571. [CrossRef]

10. Jung, D. An Investigation of the Reynolds-Number Dependence of the Axisymmetric Jet Mixing Layer Using a 138 Hot-Wire Probe and the POD. Ph.D. Thesis, State University of New York at Buffalo, Buffalo, NY, USA, 2001.

11. Berkooz, G.; Holmes, P.; Lumley, J.L. Lumley. The Proper Orthogonal Decomposition in the Analysis of Turbulent Flows. Annu. Rev. Fluid Mech. 1993, 25, 539-575. [CrossRef]

12. Jo, T.; Koo, B.; Kim, H.; Lee, D.; Yoon, J.Y. Effective sensor placement in a steam reformer using gappy proper orthogonal decomposition. Appl. Therm. Eng. 2019, 154, 419-432. [CrossRef]

13. Schmid, P.J. Dynamic mode decomposition of numerical and experimental data. J. Fluid Mech. 2010, 656, 5-28. [CrossRef]

14. Schmid, P.J. Application of the dynamic mode decomposition to experimental data. Exp. Fluids 2011, 50, 1123-1130. [CrossRef]

15. Kou, J.; Zhang, W. Dynamic mode decomposition and its application in fluid mechanics. Acta Aerodyn. Sin. 2018, 36, 163-179. 
16. Taira, K.; Brunton, S.L.; Dawson, S.T.; Rowley, C.W.; Colonius, T.; McKeon, B.J.; Schmidt, O.T.; Gordeyev, S.; Theofilis, V.; Ukeiley, L.S. Modal Analysis of Fluid Flows: An Overview. AIAA J. 2017, 55, 4013-4041. [CrossRef]

17. Xue, D.; Pan, C.; Li, G. Measurement of tip vortex instability frequency based on flow visualization. J. Beijing Univ. Aeronaut. Astronaut. 2016, 42, 837-843.

18. Dörfler, P.; Sick, M.; Coutu, A. Flow-Induced Pulsation and Vibration in Hydroelectric Machinery; Springer: Berlin/Heidelberg, Germany, 2013.

19. Wylie, E.B.; Streeter, V.L. Fluid Transients; FEB Press: Ann Arbor, MI, USA, 1983.

20. Chaudhry, M.H. Applied Hydraulic Transients; Van Nostrand Reinhold: New York, NY, USA, 1987.

21. Shechtman, Y.; Eldar, Y.C.; Cohen, O.; Chapman, H.N.; Miao, J.; Segev, M. Phase retrieval with application to optical imaging. IEEE Signal Process. Mag. 2015, 32, 87-109. [CrossRef]

22. International Electrotechnical Commission (IEC). Field Acceptance Tests to Determine the Hydraulic Performance of Hydraulic Turbines, Storage Pumps and Pump-Turbines; International Electrotechnical Commission (IEC): Geneva, Switzerland, 1991.

23. Roth, S.; Calmon, M.; Farhat, M.; Muench, C.; Huebner, B.; Avellan, F. Hydrodynamic damping identification from an impulse response of a vibration blade. In Proceedings of the 3rd IAHR International Meeting of the Workgroup on Cavitation and Dynamic Problems in Hydraulic Machinery and Systems, Brno, Czech Republic, 14-16 October 2009.

24. Ausoni, P. Turbulent Vortex Shedding from a Blunt Trailing Edge Hydrofoil. Ph.D. Thesis, Swiss Federal Institute of Technology Lausanne, Lausanne, Switzerland, 2009.

25. Menter, F.R.; Kuntz, M.; Langtry, R. Ten years of industrial experience with the SST turbulence model. Turbul. Heat Mass Transf. 2003, 4, 625-632.

26. Hellsten, A.; Laine, S. Extension of the k- $\omega$-SST turbulence model for flows over rough surfaces. In Proceedings of the 22nd Atmospheric Flight Mechanics Conference, New Orleans, LA, USA, 11-13 August 1997.

27. Huang, X.; Yang, W.; Li, Y.; Qiu, B.; Guo, Q.; Liu, Z. Review on the sensitization of turbulence models to rotation/curvature and the application to rotating machinery. Appl. Math. Comput. 2019, 341, 46-69. [CrossRef]

28. Celik, I.B.; Ghia, U.; Roache, P.J.; Freitas, C.J. Procedure for estimation and reporting of uncertainty due to discretization in CFD applications. J. Fluids Eng. 2008, 130, 0780017.

29. Zeng, Y.; Yao, Z.; Gao, J.; Hong, Y.; Wang, F.; Zhang, F. Numerical Investigation of Added Mass and Hydrodynamic Damping on a Blunt Trailing Edge Hydrofoil. J. Fluids Eng. 2019, 141, 0811088. [CrossRef]

30. Vu, T.C.; Nennemann, B.; Ausoni, P.; Farhat, M.; Avellan, F. Unsteady CFD Prediction of Von Karman Vortex Shedding in Hydraulic Turbine Stay Vanes; Hydro: Granada, Spain, 2007.

31. Kock, F.; Herwig, H. Local entropy production in turbulent shear flows: A high-Reynolds number model with wall functions. Int. J. Heat Mass Transf. 2004, 47, 2205-2215. [CrossRef] 